Teil 3

»Fake News « erkennen Glaubwürdigkeit einschätzen 


\title{
Quelle gut, alles gut? \\ Glaubwürdigkeitsbeurteilung im digitalen Raum
}

\author{
Stefanie Holzer \& Michael Sengl
}

Abstract

Vor dem Hintergrund der Glaubwürdigkeitsforschung sowie Erkenntnissen zur Selective Exposure und dem Hostile-Media-Effekt werden in diesem Beitrag zwei Studien zur Glaubwürdigkeitsbeurteilung im digitalen Raum vorgestellt: Die erste Studie identifiziert Heuristiken, die die Fähigkeit der korrekten Evaluation von Nachrichten fördern. Die zweite Studie fokussiert den Einfluss der Quelle sowie der Einstellungskonsistenz auf die Glaubwürdigkeitsbeurteilung. Zusammenfassend konnten das Mediennutzungsverhalten, die Nachrichtenkompetenz sowie die Einstellungskonsistenz als relevante Heuristiken bestätigt werden.

\section{Einführung}

Wie glaubwürdig sind die Aussagen von Carola Rackete, Kapitänin der zivilen Seenotrettung Sea-Watch? Oder die des rechtspopulistischen Compact-Magazins? Fragen, deren gesellschaftliche Relevanz im öffentlichen Diskurs unumstritten ist. Die individuellen Glaubwürdigkeitsbeurteilungen unterscheiden sich jedoch massiv. So handelt es sich bei der Glaubwürdigkeit eines Objekts nicht um eine inhärente Eigenschaft, sondern stets um einen Zuschreibungsprozess durch soziale Akteur*innen (Bentele, 1988). Dabei wird die Zuschreibung von Glaubwürdigkeit durch Heuristiken legitimiert (Blöbaum, 2016). Zum besseren Verständnis, weshalb die Glaubwürdigkeit von Informationen oftmals Gegenstand intensiver Diskussionen ist, beschäftigt sich die vorliegende Arbeit im Rahmen zweier empirischer Studien mit der Frage, welche Heuristiken bei der Glaubwürdigkeitsbeurteilung von Informationen im digitalen Raum zum Einsatz kommen. Die erste Studie beschäftigt sich mit Heuristiken, die zur korrekten Beurteilung von Nachrichtenquellen im digitalen Raum befähigen. Die zweite Studie widmet sich dem Einfluss der Quelle und der Einstellungskonsistenz auf 
die Glaubwürdigkeitsbeurteilung von Informationen in sozialen Netzwerken.

\section{»Fake News« im öffentlichen Diskurs}

In unserer Gesellschaft sind die Entwicklung und soziale Aneignung des Internets innerhalb von drei Jahrzehnten zur Selbstverständlichkeit geworden. Ein ausgesprochen kurzer Zeitraum, verglichen mit den radikalen Auswirkungen für die heutige Öffentlichkeit (Hohlfeld \& Strobel, 2011). So tritt »der (semi-)professionalisierte und redaktionell organisierte Journalismus « (Neuberger \& Quandt, 2010, 67) im Internet nicht mehr als Gatekeeper auf, sondern stellt vielmehr als Gatewatcher (Bruns, 2005) eine »empfehlende Orientierung und revidierbare Auswahl« (Neuberger, 2009, 49) von Informationen bereit. Das ehemals »passive[...], disperse [...] Massenpublikum « (Neuberger \& Quandt, 2010,67) hat wiederum die Selektionslogik der klassischen Massenmedien überwunden und kann mit Leichtigkeit Informationen an "potenziell unbegrenzte Publika« (Zimmermann \& Kohring, 2018, 526) kommunizieren sowie Anschlusskommunikation öffentlich artikulieren (Neuberger, 2009). Dieser vereinfachte Zugang zur Öffentlichkeit hat nicht nur quantitative, sondern auch qualitative Auswirkungen auf das verfügbare Informationsangebot (Neuberger, 2004). Unter anderem erleichtern die neuen Rahmenbedingungen digitaler und vor allem sozialer Medien nach Zimmermann und Kohring die »massenhafte Veröffentlichung falscher Tatsachenbehauptungen« $(2018,526)$.

In öffentlichen und politischen Diskussionen wird in diesem Kontext oftmals von sogenannten »Fake News « gesprochen. Auch in der Wissenschaft wurden innerhalb kurzer Zeit zahlreiche Ansätze entwickelt, diese spezielle Form von Desinformation begrifflich zu fassen (Zimmermann \& Kohring, 2018). Tandoc, Lim und Ling (2018) identifizieren in einer Literaturstudie die sechs Kategorien Nachrichtensatire, Nachrichtenparodien, Fabricated News, Bildmanipulation, Werbung und PR sowie Propaganda.

Gelfert $(2018,108)$ plädiert hingegen dafür, »echte Fake News« von Falschmeldungen, Satire und ähnlichen Formaten zu unterscheiden: »Fake news is the deliberate presentation of (typically) false or misleading claims as news, where the claims are misleading by design. « Beim Abgleich der Arten potentieller »Fake News« nach Tandoc et al. (2018) mit der Definition nach Gelfert erfüllen nur die Formate Propaganda und Fabricated News alle Kriterien. Im Folgenden wird daher für diese nachrichtlich anmutenden Formate der Begriff »Fake News« verwendet. 


\section{Verhältnis von Vertrauen und Glaubwürdigkeit}

Angesichts der Vielzahl an Kommunikatoren sowie der ungebremsten Veröffentlichung von unterschiedlichen Realitätskonstruktionen und falschen Tatsachenbehauptungen, die über »Fake News « hinaus gehen, werden Vertrauenshandlungen in der Internetöffentlichkeit zu einer unverzichtbaren Leistung. Dennoch ist die begriffliche Abgrenzung von Vertrauen und Glaubwürdigkeit oftmals unscharf oder überhaupt nicht gegeben. Im Allgemeinen wird Vertrauen als Mechanismus zur Komplexitätsreduktion verstanden (Luhmann, 2009). Grund hierfür ist das Prinzip der doppelten Kontingenz. So kennzeichnet sich der öffentliche Diskurs durch eine hohe Ungewissheit und beobachterabhängige Selektivität (Kohring, 2001). In diesem kommunikativen Umfeld ermöglichen vergangene Erfahrungen (Bentele \& Seidenglanz, 2008) den Vollzug von Vertrauenshandlungen und damit zukünftiges Anschlusshandeln (Kohring, 2001).

Ebenso wie Vertrauen beschreibt der Begriff der Glaubwürdigkeit keine inhärente Eigenschaft eines Vertrauensobjektes, sondern ist »das Ergebnis eines Zuschreibungsprozesses« (Lis \& Korchmar, 2013, 21). Konkret führt Bentele aus: »Glaubwürdigkeit läßt sich bestimmen als eine Eigenschaft, die Menschen, Institutionen und deren kommunikativen Produkten [...] von jemandem (Rezipienten) in bezug [sic] auf etwas (Ereignisse, Sachverhalte usw.) zugeschrieben wird « (Bentele, 1988, 408). Auch in der Kommunikationswissenschaft wird die Glaubwürdigkeit aus Sicht der Rezipient*innen beschrieben: »Glaubwürdigkeit substituiert fehlende objektive Beweise und stellt daher ein Informationssurrogat dar, das den Bereitschaftsgrad des Empfängers bestimmt, die von der Quelle erhaltene Information [...] zu übernehmen und ihr dabei einen bestimmten Gehalt hinsichtlich der Übereinstimmung mit der Realität zuzuschreiben« (Eisend, 2003, 64). Ein Modell der Glaubwürdigkeitszuschreibung im digitalen Raum ist das Sphärenmodell von Lucassen und Schraagen (2012), das vier verschiedene Ebenen von Glaubwürdigkeitsbeurteilungen unterscheidet:

- Glaubwürdigkeit der Information (z. B. einzelne Aussage)

- Glaubwürdigkeit der Quelle (z. B. der Publikation oder Person)

- Glaubwürdigkeit des Mediums (z. B. Zeitung oder Internet)

- Vertrauensfähigkeit (als Persönlichkeitsmerkmal)

Matthes und Kohring $(2003,11)$ untergliedern Vertrauen in die Subdimensionen »Vertrauen in die Themenselektivität «, »Vertrauen in die Faktenselektivität«, »Vertrauen in die Richtigkeit von Beschreibungen« und »Ver- 
trauen in journalistische Bewertungen «. Die Ebene der Richtigkeit von Beschreibungen wird von Matthes und Kohring auch als Glaubwürdigkeit bezeichnet. »Glaubwürdigkeit wird damit als ein Teilkonzept von Vertrauen in Journalismus verstanden« (Matthes \& Kohring, 2003, 11).

\section{Heuristiken der Glaubwürdigkeitsbeurteilung}

In der Wissenschaft wird für den Prozess der Glaubwürdigkeitsbeurteilung oftmals das Elaboration Likelihood Model (Petty \& Cacioppo, 1986) aufgegriffen (Hu \& Sundar, 2010; Maurer, Jost, Pfoh, Porath \& Wilke, 2018; Nawratil, 1999), dieses unterscheidet zwischen einer zentralen und einer peripheren Route der Informationsverarbeitung. Da die Fülle an Informationen mit der begrenzten kognitiven Kapazität des Publikums schwer vereinbar ist, gewinnen oberflächliche Beurteilungsprozesse mithilfe von Heuristiken an Bedeutung (Metzger \& Flanagin, 2013). Im Folgenden werden daher Heuristiken auf Seiten des sozialen Akteurs beziehungsweise der sozialen Akteurin sowie des Vertrauensobjekts betrachtet und als Grundlage für die Zuschreibung von Glaubwürdigkeit verstanden. Damit bezieht sich die Arbeit auf Blöbaum, welcher konstatiert, dass Heuristiken »rapid assessment[s] of trustworthiness « $(2016,11)$ erlauben und auf allen Glaubwürdigkeitsebenen angesiedelt sein können (Blöbaum, 2016). Diese Ebenen werden in Anlehnung an das Sphärenmodell von Lucassen und Schraagen (2012) untergliedert. Für die Zuschreibung von Glaubwürdigkeit wird auf die Untergliederung von Kohring (2001) als Teildimension von Vertrauen zurückgegriffen. 


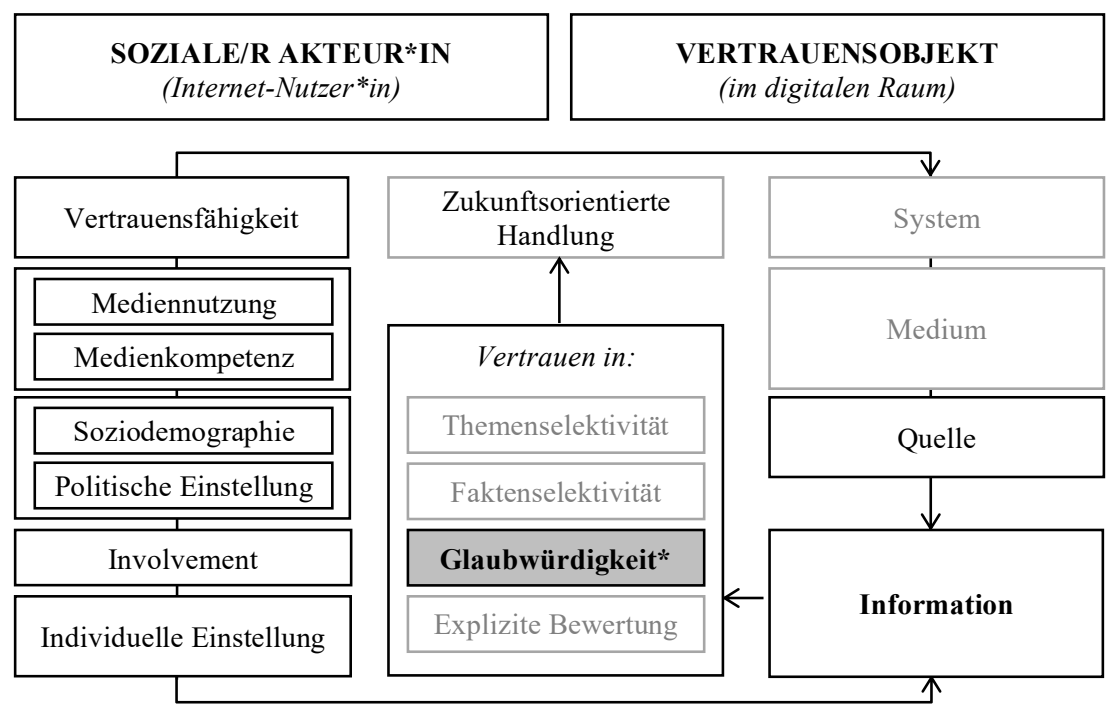

Subjektive Zuschreibung von Glaubwürdigkeit

Anmerkung: * Richtigkeit von Fakten nach Kohring (2001)

Abbildung 1: Kontingenzmodell der Glaubwürdigkeitszuschreibung (erweitertes Modell nach Blöbaum (2016))

Die psychologische Vertrauensfähigkeit ist eine wichtige Heuristik der Glaubwürdigkeitsbeurteilung auf der Akteur*innenseite, die nach Lucassen und Schraagen (2012) eine moderierende Rolle in Bezug auf die anderen Sphären der Glaubwürdigkeitsbeurteilung einnimmt. Darüber hinaus sind die Mediennutzung (Kiousis, 2001; Rimmer \& Weaver, 1987; Schranz, Schneider \& Eisenegger, 2018) sowie die Medien-/Nachrichtenkompetenz (Ashley, Maksl \& Craft, 2013; Newman, Fletcher, Kalogeropoulos, Levy \& Nielsen, 2018) zu nennen. Hier zeigen Vorstudien, dass eine Präferenz von klassischen Nachrichtenmedien in Abgrenzung zu alternativen Nachrichtenseiten (Tsfati \& Ariely, 2014; Tsfati \& Cappella, 2003) sowie eine hohe Medienkompetenz (Voigt, 2016) Rezipient*innen befähigen, die Glaubwürdigkeit von Nachrichtenquellen im digitalen Raum korrekt zu beurteilen. Auf Seiten des sozialen Akteurs beziehungsweise der sozialen Akteurin steht neben soziodemographischen Merkmalen auch die politische Orientierung der Rezipient*innen, die schon in der Vergangenheit als relevanter Einflussfaktor der Glaubwürdigkeitsbeurteilung identifiziert wurde 
(Schranz et al., 2018). Konkret stellt Lee $(2010,17)$ fest, dass »the more liberal and pro-Democrat [consumers] are, the more likely they will trust the media to report fairly« - ein Ergebnis, das auch von anderen Studien mitgetragen wird (Sängerlaub, Meier \& Rühl, 2018; Tsfati \& Cappella, 2003).

Angesichts der großen Vielfalt an Informationen im Internet, unabhängig davon, ob richtig oder falsch, erscheint zudem das Involvement sowie die individuelle Einstellung eines sozialen Akteurs beziehungsweise einer sozialen Akteurin von zunehmender Bedeutung. Aufbauend auf den Erkenntnissen zur kognitiven Dissonanz (Festinger, 2001) und zur Selective Exposure (Lazarsfeld, Berelson \& Gaudet, 1969) ergibt sich die Heuristik der Einstellungskonsistenz mit der inhaltlichen Ausrichtung der Information. Die Selective-Exposure-Theorie beschreibt dabei, dass Nutzer*innen eher diejenigen Quellen konsumieren, die ihrer Einstellung entsprechen, um kognitive Dissonanz zu vermeiden (Stroud, 2014). Metzger et al. (2015) haben festgestellt, dass Rezipient*innen diejenigen Quellen als glaubwürdiger ansehen, die mit ihrer Einstellung übereinstimmen und diese dementsprechend eher nutzen. Auch Maurer et al. $(2018,304)$ weichen von der Vorstellung ab, dass die "wahrgenommene Glaubwürdigkeit der Medienberichterstattung davon abhängt, inwieweit diese journalistischen Qualitätskriterien entspricht«. Stattdessen bestätigen sie den Einfluss der Konsistenz von Voreinstellungen auf die Glaubwürdigkeitsbeurteilung (Maurer et al., 2018). Dabei beziehen sich die Autor*innen jedoch nicht auf die Theorie der Selective Exposure, sondern den Hostile-Media-Effekt (Vallone, Ross \& Lepper, 1985), welcher ebenfalls einen Einfluss individueller Voreinstellungen auf die Beurteilung von Medieninhalten unterstellt. Eine ausgewogene mediale Berichterstattung wird demnach immer als voreingenommen angesehen, vor allem, wenn das Thema wichtig für die Rezipient*innen ist. Dabei wird unterstellt, das Medium bevorzuge die jeweilige Gegenseite (Ariyanto et al., 2007; Matthes, 2013). Der Effekt wird weiter verstärkt, wenn dem berichtenden Medium eine andere als seine eigene Ideologiezugehörigkeit zugeschrieben wird (Ariyanto, Hornsey \& Gallois, 2007).

Neben persönlichkeitsspezifischen Merkmalen wirken sich auch Heuristiken der einzelnen Vertrauensobjekte wie »message structure, message content and message delivery or presentation style« (Metzger et al., 2003, 302) auf die Glaubwürdigkeitsbeurteilung aus. Die vorliegende Arbeit rückt zudem die Ebene der Quelle in den Vordergrund. Der Grundstein zur wissenschaftlichen Auseinandersetzung mit der Quellenglaubwürdigkeit wurde mit dem zweidimensionalen Konstrukt expertness und trustworthi- 
ness von Hovland, Janis \& Kelley (1953) gelegt. Demnach gelten Quellen als glaubwürdig, wenn sie einerseits eine hohe Expertise innerhalb eines Themenbereichs aufweisen und andererseits bereit sind, eine Thematik »objektiv und unverfälscht zu kommunizieren« (Köhnken, 1990, 152). Darauf aufbauend haben sich zahlreiche Studien mit ergänzenden oder alternativen Faktoren der Quellenglaubwürdigkeit befasst (im Überblick Nawratil, 2006). Insgesamt zeigt sich: Je höher die zugeschriebene Quellenglaubwürdigkeit ist, desto glaubwürdiger werden die entsprechenden Botschaften beurteilt (Kang, Bae, Zhang \& Sundar, 2011; Sundar, Knobloch-Westerwick \& Hastall, 2007; Winter \& Krämer, 2014).

Studie 1 - Beurteilung der Glaubwürdigkeit von Nachrichtenquellen im digitalen Raum

Ziel der ersten hier vorgestellten Studie war es, zu untersuchen, welche Faktoren auf persönlicher Ebene Menschen dazu befähigen, die Glaubwürdigkeit von Nachrichtenquellen im digitalen Raum korrekt zu beurteilen.

Die der Arbeit zugrundeliegenden Hypothesen basieren auf der Literatur zu den vorgestellten Heuristiken auf Akteur*innenebene.

H1: Je liberaler die politischen Ansichten der Befragten sind, desto ausgeprägter ist die individuell-persönliche Fähigkeit, die Glaubwürdigkeit von Nachrichtenquellen im digitalen Raum korrekt zu beurteilen (basierend auf Lee, 2010; Sängerlaub et al., 2018; Schranz et al., 2018; Tsfati \& Cappella, 2003).

H2: Je wichtiger klassische Nachrichtenmedien im individuellen Nutzungsmix sind, desto ausgeprägter ist die individuell-persönliche Fähigkeit, die Glaubwürdigkeit von Nachrichtenquellen im digitalen Raum korrekt zu beurteilen (basierend auf Kiousis, 2001; Rimmer \& Weaver, 1987; Schranz et al., 2018; Tsfati \& Ariely, 2014; Tsfati \& Cappella, 2003).

H3.1: Je schwächer die Verbindung der Rezipient*innen zum Thema, desto ausgeprägter ist die individuell-persönliche Fähigkeit, die Glaubwürdigkeit von Nachrichtenquellen im digitalen Raum korrekt zu beurteilen (basierend auf Ariyanto et al., 2007; Matthes, 2013).

H3.2: Je geringer die Differenz zwischen der Ausrichtung des Mediums und der Ideologie der Rezipient*innen ist, desto ausgeprägter ist die indivi- 
duell-persönliche Fähigkeit, die Glaubwürdigkeit von Nachrichtenquellen im digitalen Raum korrekt zu beurteilen (basierend auf Ariyanto et al., 2007; Matthes, 2013).

H4: Je größer die Nachrichtenkompetenz der Befragten, desto ausgeprägter ist die individuell-persönliche Fähigkeit, die Glaubwürdigkeit von Nachrichtenquellen im digitalen Raum korrekt zu beurteilen (basierend auf Ashley et al., 2013; Newman et al., 2018; Voigt, 2016).

H5: Je höher das Vertrauen der Befragten in das Medium Internet, desto ausgeprägter ist die individuell-persönliche Fähigkeit, die Glaubwürdigkeit von Nachrichtenquellen im digitalen Raum korrekt zu beurteilen (basierend auf Lucassen \& Schraagen, 2012).

H6: Je höher die allgemeine Vertrauensfähigkeit der Befragten, desto ausgeprägter ist das Vertrauen der Befragten in das Medium Internet (basierend auf Lucassen \& Schraagen, 2012).

H7: Je höher das Vertrauen der Befragten in den Journalismus, desto ausgeprägter ist die individuell-persönliche Fähigkeit, die Glaubwürdigkeit von Nachrichtenquellen im digitalen Raum korrekt zu beurteilen (basierend auf Kohring \& Matthes, 2004; Matthes \& Kohring, 2003).

Um diese Hypothesen zu überprüfen, wurde im Juli 2018 eine quantitative Online-Befragung durchgeführt. Den Teilnehmer*innen wurden dabei drei reale Artikel von Online-Medien vorgelegt. Der erste Text zur russischen Präsidentschaftswahl stammt von RT Deutsch (2018), einem staatsnahen russischen Medium, das mehrfach für seine tendenziöse Berichterstattung kritisiert wurde (Kohrs, 2017; Luther, 2014; Schulze, 2017). Der Artikel selbst ist ebenfalls inhaltlich unausgewogen und klar der Kategorie Propaganda zuzuordnen. Der zweite Artikel behandelt das Thema Migration und stammt aus dem rechten Nachrichtenportal Watergate.tv (Watergate Redaktion, 2018). Er ist den Fabricated News zuzuordnen. Der Text ist sprachlich fragwürdig und inhaltlich sowohl inkonsistent als auch falsch. Vom Recherchezentrum Correctiv wurde er als »Level-5-Fake News« eingestuft, was das obere Ende der Skala abbildet (Schmüser, 2018). Der dritte Artikel zum Pflegenotstand in Deutschland ist eine hochwertige journalistische Nachricht des Tagesspiegels (Heine, 2018), der als eine der besten deutschen Tageszeitungen gilt (Wellbrock, 2011) und sollte testen, ob die Rezipient*innen Qualitätsjournalismus auch als solchen erkennen. 
Nach dem Lesen der Artikel sollten die Befragten diese jeweils auf ihre Glaubwürdigkeit beurteilen und ihre persönliche Einstellung zu den behandelten Sachverhalten angeben. Weiterhin wurden im Rahmen des Fragebogens die politische Orientierung, die Vertrauensebenen nach Lucassen und Schraagen (2012) sowie das Vertrauen in den Journalismus nach Matthes und Kohring (2004; 2003), die Mediennutzung, die Nachrichtenkompetenz sowie demographische Angaben erhoben.

Insgesamt wurden 197 Fragebögen vollständig ausgefüllt. Das Durchschnittsalter der Teilnehmer*innen lag bei 34,020 Jahren $(S D=15.896)$. Die Befragten waren überdurchschnittlich gut gebildet $(66,0 \%(n=130)$ besitzen die (Fach-)Hochschulreife) und sind zu fast gleichen Teilen männlich $(47,7 \% ; n=94)$ und weiblich $(51,8 \% ; n=102)$. Alle Befragten hatten zum Zeitpunkt der Erhebung einen Internetzugang, konsumierten OnlineNachrichten und waren deutsche Staatsbürger*innen.

H1 bezieht sich auf den Einfluss der (partei-)politischen Orientierung der Befragten auf die Fähigkeit, die Glaubwürdigkeit von Nachrichtenquellen korrekt zu beurteilen. Diese Fähigkeit wurde operationalisiert, indem die Befragten drei journalistische Artikel als Stimulusmaterial bewerteten. Dazu wurden ihnen Fragen in Anlehnung an Kohring und Matthes (2004) gestellt. Je höher der gleichgerichtete Mittelwert der Antworten über alle Artikel hinweg, desto korrekter die Glaubwürdigkeitsbeurteilung. Die politische Orientierung wurde über eine Selbsteinschätzung auf der politischen Skala von links bis rechts sowie eine sogenannte Sonntagsfrage nach der parteipolitischen Präferenz operationalisiert. Zwar zeigt sich durchaus eine Tendenz, dass eine rechte politische Orientierung mit einer geringeren Fähigkeit zur Glaubwürdigkeitsbeurteilung zusammenhängt (sowohl bei der Skala links-rechts als auch als Ausreißer bei einer parteipolitischen Präferenz der AfD), beide Korrelationen sind aber nicht signifikant (links-rechts: $r=-.116, p=.134, n=168$; parteipol.: $(F(6,185)=1.226, p=.295)$.

Für die Prüfung der zweiten Hypothese sollten die Befragten verschiedene Arten von Online-Nachrichtenangeboten entsprechend ihres individuellen Nutzungsmixes auswählen und nach Wichtigkeit anordnen. Signifikante Korrelationen ergeben sich nur bei zwei Angebotstypen: Zeitungen und alternativen Quellen. Hypothesengemäß bedingt ein hoher Stellenwert der Online-Angebote klassischer Zeitungen eine signifikant ausgeprägtere Fähigkeit, Nachrichtenquellen korrekt auf ihre Glaubwürdigkeit zu beurteilen $(r=.187, p=.009, n=197)$. 


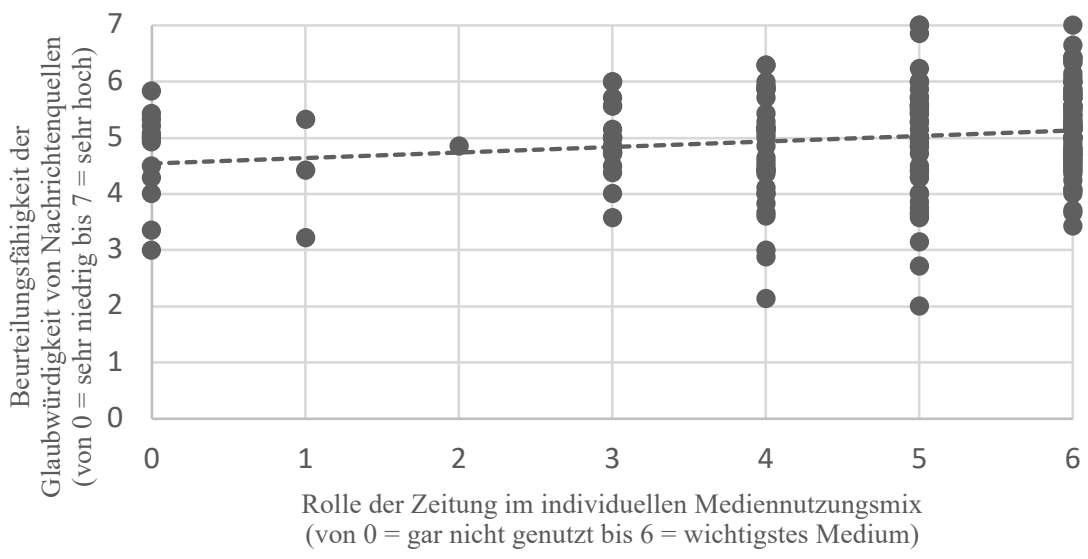

Abbildung 2: Zusammenhang von Zeitungspräferenz und Fähigkeit zur Beurteilung der Glaubwürdigkeit von Nachrichtenquellen

Ein gegensätzliches Resultat ergibt sich beim Stellenwert von alternativen Nachrichtenquellen. Hier bedingt ein höherer Wert eine signifikant niedrigere Fähigkeit zur Glaubwürdigkeitsbeurteilung $(r=-.200, p=.005, n=$ 197).

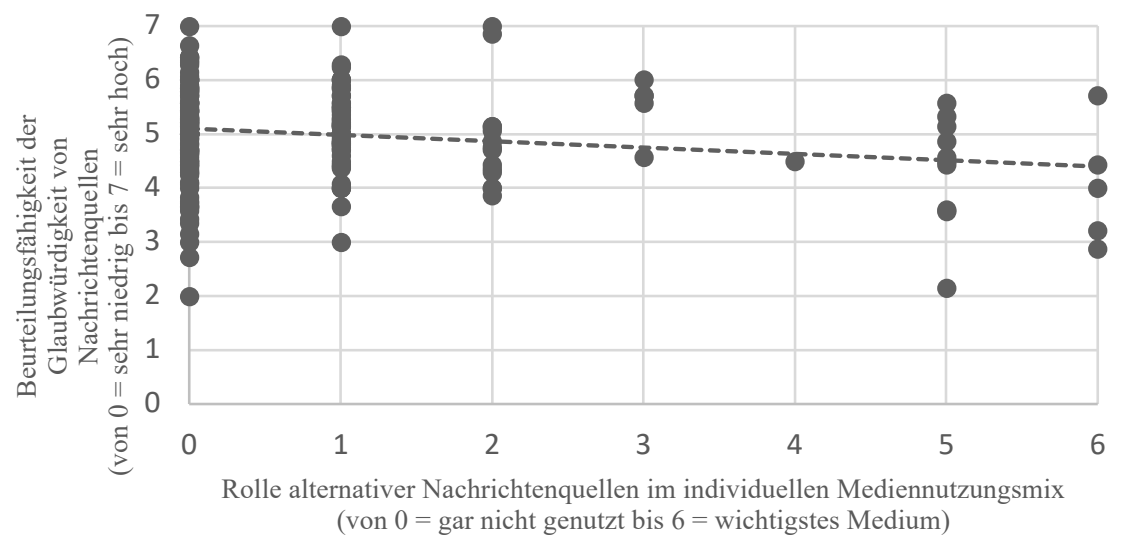

Abbildung 3: Zusammenhang der Präferenz von alternativen Nachrichtenmedien und Fähigkeit zur Beurteilung der Glaubwürdigkeit von Nachrichtenquellen 
Auch wenn die übrigen Nachrichtenquellen keine signifikanten Ergebnisse liefern, lassen diese beiden Extrema dennoch darauf schließen, dass ein $\mathrm{Zu}-$ sammenhang besteht, $\mathrm{H} 2$ wird damit angenommen.

Die Befragten sollten für H3.1 nach der Glaubwürdigkeitsbeurteilung der einzelnen Artikel jeweils einschätzen, wie wichtig ihnen die Themen persönlich sind. Dabei ist lediglich beim Thema Pflegenotstand ein signifikanter Zusammenhang zur Glaubwürdigkeitsbeurteilung des Artikels festzustellen $(r=.178, p=.014, n=190)$. Die Hypothese wird damit verworfen.

Zusammen mit der zuvor geschilderten Einordnung der Wichtigkeit der jeweiligen Themen sollten die Befragten ihre persönliche Einstellung zu den Themen der Artikel angeben. Hierbei zeigt sich ein eindeutiges Ergebnis: Je eher die persönliche Meinung der Befragten der Ausrichtung des jeweiligen Artikels entspricht, desto glaubwürdiger schätzen sie diesen ein. Dieser Effekt zeigt sich deutlich bei allen drei Themen Migrationspolitik ( $r$ $=.414, p<.001, n=177)$, russische Präsidentschaftswahl $(r=.219, p=$ $.005, n=163)$ sowie Pflegenotstand $(r=.239, p=.001, n=183)$. Auch wenig glaubwürdige Artikel werden bei Einstellungskonsistenz als glaubwürdiger eingeschätzt. H3.2 wird damit verworfen. Der Hostile-Media-Effekt, der genau diesen Zusammenhang voraussagt, ist in der Untersuchung eindeutig nachweisbar.

Den Befragten wurden drei Wissensfragen zur Finanzierung des öffentlich-rechtlichen Rundfunks, der Urheberschaft von Pressemitteilungen und der Nachrichtenausspielung in den sozialen Medien gestellt, deren gleichgerichteter Mittelwert als Maß für die Nachrichtenkompetenz genutzt wird. Der Zusammenhang mit der Beurteilungsfähigkeit der Glaubwürdigkeit ist signifikant positiv ( $r=.336, p<.001, n=194)$, H4 kann damit angenommen werden.

In Bezug auf das Vertrauen der Befragten in das Medium Internet kann kein signifikanter Zusammenhang nachgewiesen werden $(r=.062, p=.395$, $n=193)$, H5 wird verworfen. Gleiches gilt für die Vertrauensfähigkeit ( $r=$ -.038, $p=.603, n=194$ ), auch H6 muss daher abgelehnt werden.

Für die Beantwortung von $\mathrm{H} 7$ wurde untersucht, ob das Vertrauen in den Journalismus sich auf die Beurteilungsfähigkeit der Glaubwürdigkeit auswirkt. Für das Gesamtkonstrukt muss diese Hypothese verworfen werden $(r=.101, p=.163, n=193)$. Da dieses Gesamtvertrauen nach Matthes und Kohring (2004; 2003) operationalisiert wurde, lassen sich auch die Subdimensionen analysieren. Hier ist auffällig, dass sowohl das Vertrauen in explizite Bewertungen $(r=.154, p=.033, n=191)$ als auch insbesondere das Vertrauen in die Richtigkeit von Beschreibungen $(r=.196, p=.009$, $n=177)$ signifikant mit der Beurteilungsfähigkeit korrelieren. Je eher 
Rezipient*innen also der Meinung sind, dass die berichteten Fakten der subjektiv empfundenen Wahrheit entsprechen und dass zu einem Thema alle wichtigen Fakten genannt werden (Glaubwürdigkeit nach Kohring und Matthes (2003)), desto eher sind sie in der Lage, die Glaubwürdigkeit von Nachrichtenquellen im digitalen Raum korrekt zu beurteilen.

Bei den soziodemographischen Faktoren wiesen weder Geschlecht $(t(191)=.051, p=.959)$, Alter $(r=-.128, p=.076, n=194)$ noch Bildung $(F(7,186)=.423, p=.887)$ einen signifikanten Einfluss auf die Beurteilungsfähigkeit auf.

Ziel der Untersuchung war es, herauszufinden, welche Faktoren auf individuell-persönlicher Ebene die Fähigkeit beeinflussen, die Glaubwürdigkeit von Nachrichtenquellen im digitalen Raum korrekt zu beurteilen. Als relevant konnten die Faktoren der Mediennutzungspräferenz, Nachrichtenkompetenz und Teile des Vertrauens in den Journalismus identifiziert werden.

Um den Fragebogen kompakt zu halten, wurde das Konstrukt der Medienkompetenz auf den Teilaspekt der Nachrichtenkompetenz komprimiert und eine ausführliche Itembatterie durch drei Wissensfragen ersetzt. Vor dem Hintergrund, dass der Einfluss der Nachrichtenkompetenz sich als eines der signifikanten Ergebnisse herausgestellt hat, bietet es sich hier an, den Einfluss in Folgestudien detaillierter zu prüfen. Gleiches gilt für die Erfassung der Mediennutzung.

Rezipient*innen mit einem hohen Grad an Nachrichtenkompetenz, also fundiertem Wissen über die Rahmenbedingungen, Strukturen und Akteur*innen unseres Mediensystems, sowie einem Mediennutzungsverhalten, bei dem qualitativ hochwertige Nachrichtenquellen präferiert werden, sind eher in der Lage, die Glaubwürdigkeit einer Nachrichtenquelle korrekt beurteilen zu können. Dieses Bewusstsein gilt es zu steigern, um die Verbreitung und Wirkung von »Fake News« einzudämmen.

\section{Studie 2 - Einfluss von Quelle und Einstellungskonsistenz auf die Glaub-} würdigkeitsbeurteilung von Informationen in sozialen Netzwerken

Die zweite Studie widmet sich dem Einfluss der Quelle sowie der Einstellungskonsistenz der Rezipient*innen mit Informationen in sozialen Netzwerken auf deren Glaubwürdigkeitsbeurteilung. Dabei geht es nicht um die Fähigkeit der Rezipient*innen, den Wahrheitsgehalt einer Information tatsächlich richtig zu bestimmen, sondern vielmehr um den Einfluss einzelner 
Heuristiken auf die Zuschreibung von Glaubwürdigkeit und damit die empfundene Richtigkeit von Fakten (Kohring, 2001).

Die Wahl der Quelle als Urteilsheuristik ist auf die erhöhte Anzahl an Kommunikator*innen im Internet zurückzuführen. Aufgrund der Disintermediation durch Vertreter*innen partikularer Interessen wird hierbei insbesondere die wahrgenommene trustworthiness (Hovland et al., 1953) einer Quelle betrachtet. Metzger und Flanagin übertragen die entsprechenden Erkenntnisse aus der durch traditionelle Massenmedien hergestellten Öffentlichkeit auf die Online-Umgebung und skizzieren die "persuasive intent heuristic« (Metzger \& Flanagin, 2013, 216). Nach dieser wirkt es sich negativ auf die Glaubwürdigkeit aus, wenn soziale Rollen, die auf ein interessengeleitetes Kommunikationsverhalten hinweisen, erkennbar sind.

H1: In sozialen Netzwerken werden Informationen von gemeinwohlorientierten Quellen glaubwürdiger eingestuft als Informationen von interessengeleiteten Quellen.

Die Heuristik der Einstellungskonsistenz beruht hingegen auf der Forschung zu Selective-Exposure-Effekten (Lazarsfeld et al., 1969) sowie der daraus abgeleiteten self confirmation heuristic nach Metzger \& Flanagin (2013).

H2: In sozialen Netzwerken werden einstellungskonsistente Informationen glaubwürdiger eingestuft als einstellungsinkonsistente Informationen.

Die theoretische Fundierung sowie der aktuelle Forschungsstand legen nahe, dass die Glaubwürdigkeitsbeurteilung einer Information immer auf dem Zusammenspiel verschiedener Heuristiken beruht (Haas \& Unkel, 2015; Sundar et al., 2007; Tandoc, 2019).

H3: Je mehr positive Glaubwürdigkeitsindikatoren vorhanden sind, desto höher ist die zugeschriebene Glaubwürdigkeit von Informationen in sozialen Netzwerken.

Durch die Kombination der Quelle sowie der Einstellungskonsistenz stellt sich zudem die Frage, welche der Heuristiken die größere Relevanz hat, sofern sich die Heuristiken widersprechen (Winter, 2013). Aufgrund der ambivalenten Forschungsergebnisse und dem Mangel an äquivalenten Forschungsfragen wird diese Forschungsfrage explorativ gestellt: 
FF1: Hat bei Widerspruch der Glaubwürdigkeitsindikatoren die Quelle oder die Einstellungskonsistenz mehr Einfluss auf die Glaubwürdigkeitsbeurteilung von Informationen in sozialen Netzwerken?

Zur Überprüfung der Hypothesen wurde im April 2018 ein experimentelles Studiendesign auf SoSci Survey erstellt. Die Studie bestand aus einem 2x2between-subject-Design (gemeinwohlorientierte versus interessengeleitete Quelle sowie pro versus contra Information). Allerdings ergaben sich die finalen Experimentalgruppen erst durch den Abgleich der Pro- oder ContraVersion mit der Einstellung der Proband*innen, welche zu Beginn der Erhebung abgefragt wurde. Neben der Abfrage der Einstellung der Proband*innen anhand einer Multi-Item-Skala in Anlehnung an Wohlt et al. (2017) stellte die Glaubwürdigkeitsbeurteilung der Information einen zentralen Bestandteil der Studie dar. Da sich nicht nur journalistische Inhalte, sondern auch Informationen von interessengeleiteten Akteur*innen hinsichtlich der Richtigkeit von Fakten beurteilen lassen, basierte die entsprechende Skala in weiten Teilen auf der revidierten und validierten Skala zur Erfassung von Vertrauen in Journalismus von Kohring und Matthes (2004). Allerdings mussten im Kontext sozialer Netzwerke kleine Änderungen am Wortlaut vorgenommen werden. Zusätzlich wurden relevante Drittvariablen wie Involvement oder Soziodemographie abgefragt.

Als Stimulus dienten insgesamt vier fiktive Facebook-Beiträge. Die Wahl der Quellen fiel auf die Süddeutsche Zeitung als gemeinwohlorientierte Quelle und den Arbeitgeberverband Gesamtmetall als interessengeleitete Quelle. Auswahlkriterium war jeweils, dass die Quelle allgemein bekannt ist und eine Facebook-Präsenz aufweist. Außerdem durfte die Positionierung der Quellen zum Inhalt des Beitrags nicht allzu naheliegend sein. Für den Inhalt der Information wurde jeweils die Geflüchtetendebatte aufgegriffen, konkret die Eignung von jungen Geflüchteten als Nachwuchskräfte im deutschen Handwerk. Grund hierfür war die Notwendigkeit eines kontroversen Themas, welches sowohl die inhaltliche Ausrichtung des Beitrags als auch die Einteilung der Probanden in pro und contra zuließ. Außerdem stellt sich nach Sängerlaub et al. (2018) bei dem Thema Geflüchtete verstärkt die Frage nach der Richtigkeit der Fakten.

Der endgültige Datensatz umfasste 428 Interviews. Die Mehrheit der Teilnehmer war weiblich (67,1\%). Das durchschnittliche Alter betrug 27 Jahre $(S D=10.56)$. 49,6 Prozent der Teilnehmer*innen besaßen die allgemeine Hochschulreife und 37,6 Prozent hatten bereits einen Fachhochschuloder Hochschulabschluss. Über die Hälfte der Teilnehmer*innen (64,2 \%) gaben das Internet als wichtigste Informationsquelle an. Innerhalb des Inter- 
nets nutzten 23,1 Prozent der Teilnehmer*innen soziale Netzwerke täglich als Informationsquelle für das aktuelle Tagesgeschehen; 22,4 Prozent mehrmals pro Woche. Allerdings ist zu berücksichtigen, dass die Stichprobe nicht repräsentativ für die Gesamtbevölkerung Deutschlands ist.

Im Rahmen der nachträglichen Zuordnung der inhaltlichen Ausrichtung des Facebook-Beitrags und der Einstellung der Proband*innen wurden zwei Kontrollgruppen »Neutral/Gemeinwohlorientiert « und »Neutral/Interessengeleitet « gebildet, da insgesamt 66 Proband*innen eine neutrale Einstellung zur Geflüchtetendebatte aufwiesen. Die restlichen 362 Proband*innen verteilten sich in vergleichbaren Anteilen auf die restlichen Experimentalgruppen. Anhand dieser wurde zunächst der Haupteffekt der Quelle auf die Glaubwürdigkeitsbeurteilung von Informationen in sozialen Netzwerken berechnet. Aufgrund der insgesamt sehr hohen Reliabilität der verwendeten Skala zur Messung der Glaubwürdigkeit (Cronbach's $\alpha=$.937) wurde die abhängige Variable der Glaubwürdigkeit anhand des Mittelwerts aus allen Items der Skala untersucht. Die Homogenität der Varianzen wurde mithilfe des Levene-Tests geprüft $(F(3,358)=.722, p=.510)$. Werden die deskriptiven Statistiken der zweifaktoriellen Varianzanalyse betrachtet, scheint es, dass Informationen von gemeinwohlorientierten Quellen $(M=3.098, S D=$ $1.034, n=181)$ glaubwürdiger eingestuft werden als Informationen von interessengeleiteten Quellen $(M=2.949, S D=1.043, n=181)$. Allerdings ist dieser Mittelwertunterschied auf Basis des zweiseitigen Signifikanzniveaus von $p<.05$ nicht signifikant $\left(F(1,358)=2.883, p=.090, \eta^{2}=.008\right)$. Die erste Hypothese muss daher verworfen werden. Bei der zweiten Hypothese wurde die alleinige Wirkung der Einstellungskonsistenz oder -inkonsistenz betrachtet. Bereits die deskriptive Statistik zeigt, dass einstellungskonsistente Informationen $(M=3.541, S D=.888, n=172)$ glaubwürdiger beurteilt wurden als einstellungsinkonsistente Informationen $(M=2.555, S D=$ $.943, n=190)$. Dieser Unterschied ist hoch signifikant $(F(1,358)=105.039$, $p<.001, \eta^{2}=.227$ ). Folglich kann die Heuristik der Einstellungskonsistenz als Glaubwürdigkeitsindikator bei Informationen in sozialen Netzwerken bestätigt werden. Der ordinale Interaktionseffekt zwischen der Heuristik der Quelle und der Einstellungskonsistenz ist wiederum nicht signifikant $\left(F(1,358)=.528, p=.468, \eta^{2}=.001\right)$. Die dritte Hypothese wird somit abgelehnt. 

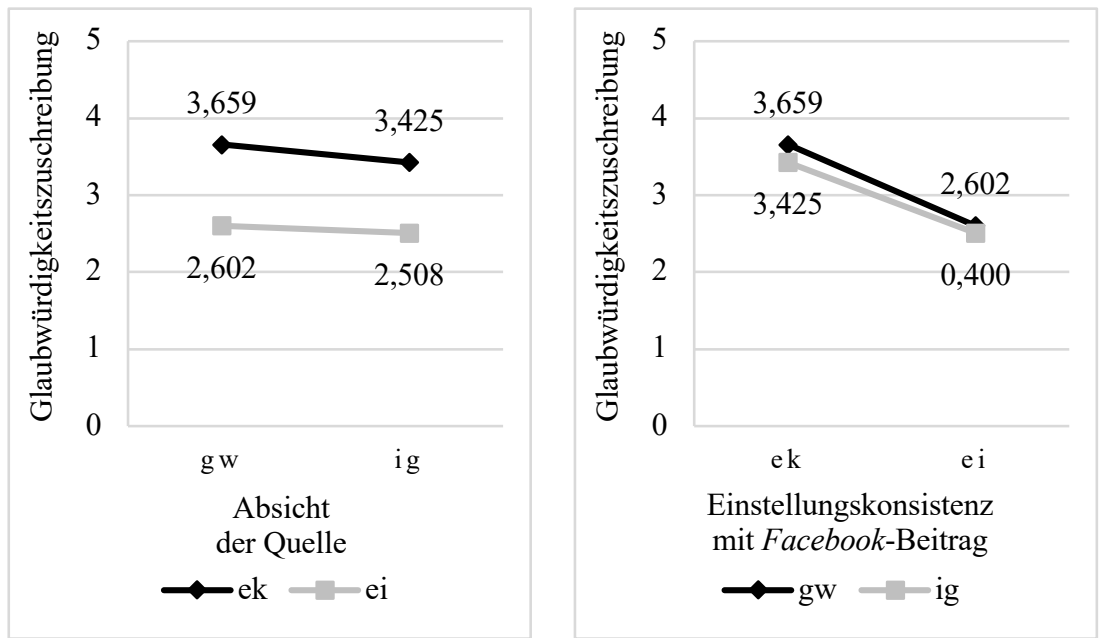

Anmerkung: ek = einstellungskonsistent, $\mathrm{ei}=$ einstellungsinkonsistent, $\mathrm{gw}=$ gemeinwohlorientiert, ig = interessengeleitet

Abbildung 4: Globale Interpretation von Haupteffekten der Quelle und der Einstellungskonsistenz, Interaktionseffekt nicht signifikant

Die explorative Forschungsfrage wurde anhand der Experimentalgruppen »Einstellungskonsistent/Interessengeleitet « $(M=3.425, S D=.923, n=87)$ und »Einstellungsinkonsistent/Gemeinwohlorientiert« $(M=2.602, S D=$ $.934, n=96)$ untersucht. Die Ergebnisse der einfaktoriellen Varianzanalyse bestätigen dabei, was sich bereits zuvor abgezeichnet hat: Obwohl beide Experimentalgruppen jeweils einen positiven und einen negativen Glaubwürdigkeitsindikator beinhalten, wird die Glaubwürdigkeit der Information nicht identisch beurteilt. Vielmehr beeinflusst die Einstellungskonsistenz die Glaubwürdigkeitsbeurteilung bei Widerspruch der Heuristiken mehr als die Quelle $\left(F(1,181)=35.881, p<.001, \eta^{2}=.165\right)$.

Angesichts des überraschenden insignifikanten Haupteffekts der Quelle ist dieses Ergebnis jedoch kritisch zu betrachten. Zwar ist es möglich, dass der Kontext sozialer Netzwerke den Einfluss der Quelle im Vergleich zu Websites und Nachrichtenaggregatoren oder klassischen Massenmedien verringert, allerdings deutet der Manipulationscheck der Quelle darauf hin, dass die Manipulation der Quelle zu unauffällig war. Das würde bedeuten, dass trotz der erkennbaren Tendenz des Effekts kein ausgeprägter Widerspruch zwischen Heuristiken herrschte und lediglich die Heuristik der Einstellungskonsistenz zum Tragen kam. Statistisch auffällig ist indessen, dass die Manipulation der Quelle in den neutralen Kontrollgruppen eine signifikante Wirkung zeigt $(t(64)=2.061, p=.043)$. Berechnungen zu den 
erhobenen Drittvariablen ergaben hingegen keinen signifikanten Einfluss auf die vorliegenden Haupteffekte. Die Studie bestätigt somit die Annahme, dass das Konzept der Selective Exposure (Stroud, 2014) nicht nur auf die Selektion von Informationen, sondern auch auf deren Glaubwürdigkeitsbeurteilung angewendet werden kann. Ob die Quelle bei der Glaubwürdigkeitsbeurteilung von Informationen in sozialen Netzwerken und im Zusammenspiel mit der Einstellungskonsistenz tatsächlich keine Wirkung hat, ist jedoch in weiteren empirischen Studien zu prüfen.

\section{Diskussion}

Diese Ergebnisse beantworten nicht nur die übergeordnete Forschungsfrage nach den Heuristiken der Glaubwürdigkeitsbeurteilung von Informationen im digitalen Raum, sondern auch die kritische Frage »Quelle gut, alles gut? « So zeigt sich eindeutig, dass die Quelle nicht allein über die Glaubwürdigkeit von Informationen in sozialen Netzwerken entscheidet. Stattdessen wirken eine Vielzahl weiterer Faktoren auf die Glaubwürdigkeitsbeurteilung ein. Zunächst konnten die Mediennutzungspräferenzen der Rezipient*innen als relevanter Faktor identifiziert werden: Je wichtiger klassische Nachrichtenmedien wie die Zeitung im individuellen Mediennutzungsmix und je unwichtiger sogenannte alternative Nachrichtenquellen sind, desto eher sind Nutzer*innen in der Lage, die Glaubwürdigkeit von Nachrichtenquellen online korrekt zu beurteilen. ${ }^{1}$ Gleiches gilt, je höher deren Nachrichtenkompetenz ist. ${ }^{1}$ Als weiterer relevanter Faktor konnte die Einstellungskonsistenz sowohl der Quelle ${ }^{1}$ als auch der Information ${ }^{2}$ mit den persönlichen Ansichten der Rezipient*innen nachgewiesen werden. Die Reichweite dieser Ergebnisse muss unter dem Gesichtspunkt bewertet werden, dass die beiden Studien zwar thematisch ähnlich und zur gleichen Zeit, ansonsten jedoch unabhängig voneinander durchgeführt wurden.

Nichtsdestotrotz stehen die Ergebnisse sowohl mit den Vorstudien zum Thema Selective Exposure als auch mit Maurer et al. (2018) in Einklang, die den Einfluss des Hostile-Media-Effekts betonen.

Somit stützen die Ergebnisse der beiden empirischen Studien die Annahme, dass die potentielle Übereinstimmung von »Fake News« mit den Voreinstellungen bestimmter Rezipient*innen zu deren Glaubwürdigkeit und damit Verbreitung beiträgt. Gerade aus dem positiven Einfluss der Rezeption qualitativ hochwertiger Nachrichten und einer gesteigerten Nach- 
richtenkompetenz lässt sich die klare Empfehlung ableiten, Medienkompetenz in der Bevölkerung weiter zu fördern und damit einen Beitrag gegen die Verbreitung und Wirkung von »Fake News« zu leisten.

\section{Literatur}

Ariyanto, Amarina; Hornsey, Matthew J. \& Gallois, Cindy (2007). Group Allegiances and Perceptions of Media Bias: Taking Into Account Both the Perceiver and the Source. Group Processes \& Intergroup Relations, 10(2), 266-279. DOI: 10.1177/1368430207074733.

Ashley, Seth; Maksl, Adam \& Craft, Stephanie (2013). Developing a News Media Literacy Scale. Journalism \& Mass Communication Educator, 68(1), 7-21. DOI: 10.1177/1077695812469802.

Bentele, Günter (1988). Der Faktor Glaubwürdigkeit. Forschungsergebnisse und Fragen für die Sozialisationsperspektive. Publizistik, 33(2/3), 406-426.

Bentele, Günter \& Seidenglanz, René (2008). Trust and Credibility - Prerequisites for Communication Management. In: Zerfass, A.; B. van Ruler \& K. Sriramesh (Hrsg.), Public Relations Research (49-62). VS Verlag für Sozialwissenschaften. DOI: 10.1007/978-3-531-90918-9_4.

Blöbaum, Bernd (2016). Key Factors in the Process of Trust. On the Analysis of Trust under Digital Conditions. In: Blöbaum, B. (Hrsg.), Trust and Communication in a Digitized World (3-25). Springer International Publishing. DOI: 10.1007/978-3-31928059-2_1.

Bruns, Axel (2005). Gatewatching: Collaborative online news production. Peter Lang Publishing.

Eisend, Martin (2003). Glaubwürdigkeit in der Marketingkommunikation: Konzeption, Einflussfaktoren und Wirkungspotenzial. Springer Fachmedien.

Festinger, Leon (2001). A Theory of Cognitive Dissonance. Stanford University Press.

Gelfert, Axel (2018). Fake News: A Definition. Informal Logic, 38(1), 84-117. DOI: 10.22329/il.v38i1.5068.

Haas, Alexander \& Unkel, Julian (2015). Glaubwürdigkeit und Selektion von Suchergebnissen. Der Einfluss von Platzierung, Reputation, Neutralität und sozialen Empfehlungen bei der Nutzung von Suchmaschinen. Medien \& Kommunikationswissenschaft, 63(3), 363-382. DOI: 10.5771/1615-634X-2015-3-363.

Heine, Hannes (2018). Pflegenotstand: Offensiver gegen den Mangel. Der Tagesspiegel, 03. Juli 2018. Abgerufen von https://www.tagesspiegel.de/politik/pflegenotstandoffensiver-gegen-den-mangel/22764090.html.

Hohlfeld, Ralf \& Strobel, Matthias (2011). Neue Medien - Neue Öffentlichkeit(en). Die Medien- und Kommunikationskonvergenz als zentrale Herausforderung der Kommunikationswissenschaft. In: Institut für interdisziplinäre Medienforschung (IfIM) (Hrsg.), Medien und Wandel (15-39). Logos-Verlag. 
Hovland, Carl I.; Janis, Irving L. \& Kelley, Harold H. (1953). Communication and Persuasion. Psychological Studies of Opinion Change. Yale University Press.

$\mathrm{Hu}$, Yifeng \& Sundar, S. Shyam (2010). Effects of Online Health Sources on Credibility and Behavioral Intentions. Communication Research, 37(1), 105-132. DOI: 10.1177/0093650209351512.

Kang, Hyunjin; Bae, Keunmin; Zhang, Shaoke \& Sundar, S. Shyam (2011). Source Cues in Online News: Is the Proximate Source More Powerful than Distal Sources? Journalism \& Mass Communication Quarterly, 88(4), 719-736. DOI: 10.1177/ 107769901108800403.

Kiousis, Spiro (2001). Public Trust or Mistrust? Perceptions of Media Credibility in the Information Age. Mass Communication and Society, 4(4), 381-403. DOI: 10.1207/S15327825MCS0404_4.

Köhnken, Günter (1990). Glaubwürdigkeit: Untersuchungen zu einem psychologischen Konstrukt. Psychologie Verlags Union.

Kohring, Matthias (2001). Vertrauen in Medien - Vertrauen in Technologie. DOI: 10.18419/opus-8677.

Kohring, Matthias \& Matthes, Jörg (2004). Revision und Validierung einer Skala zur Erfassung von Vertrauen in Journalismus. Medien \& Kommunikationswissenschaft, 52(3), 377-385. DOI: 10.5771/1615-634x-2004-3-377.

Kohrs, Camilla (2017). Russische Propaganda für deutsche Zuschauer. Die Medien der Neuen Rechten, Teil 7: Das Webportal »RT Deutsch«. Correctiv, 04. Januar 2017. Abgerufen von https://correctiv.org/aktuelles/neue-rechte/2017/01/04/russischepropaganda-fuer-deutsche-zuschauer.

Lazarsfeld, Paul Felix; Berelson, Bernard \& Gaudet, Hazel (1969). The people's choice: How the voter makes up his mind in a presidential campaign. Columbia University Press.

Lee, Tien-Tsung (2010). Why They Don't Trust the Media: An Examination of Factors Predicting Trust. American Behavioral Scientist, 54(1), 8-21. DOI: 10.1177/0002764210376308.

Lis, Bettina \& Korchmar, Simon (2013). Digitales Empfehlungsmarketing: Konzeption, Theorien und Determinanten zur Glaubwürdigkeit des Electronic Word-of-Mouth (EWOM). Springer Gabler.

Lucassen, Teun \& Schraagen, Jan Maarten (2012). Propensity to trust and the influence of source and medium cues in credibility evaluation. Journal of Information Science, 38(6), 566-577. DOI: 10.1177/0165551512459921.

Luhmann, Niklas (2009). Vertrauen: Ein Mechanismus der Reduktion sozialer Komplexität. Lucius \& Lucius.

Luther, Carsten (2014). RT Deutsch: Das hat uns gerade noch gefehlt. Zeit Online, 19. November 2014. Abgerufen von https:/www.zeit.de/politik/ausland/2014-11/rtdeutsch-russland-propaganda-luegen.

Matthes, Jörg (2013). The Affective Underpinnings of Hostile Media Perceptions: Exploring the Distinct Effects of Affective and Cognitive Involvement. Communication Research, 40(3), 360-387. DOI: 10.1177/0093650211420255. 
Matthes, Jörg \& Kohring, Matthias (2003). Operationalisierung von Vertrauen in Journalismus. Medien \& Kommunikationswissenschaft, 51(1), 5-23. DOI: 10.5771/1615634x-2003-1-5.

Maurer, Marcus; Jost, Pablo; Pfoh, Milan; Porath, Maximiliane \& Wilke, Lea (2018). Motivierte Zweifel. Wie die Voreinstellungen der Rezipienten zum Berichterstattungsgegenstand ihre Wahrnehmung der Medienglaubwürdigkeit beeinflussen. $M e-$ dien \& Kommunikationswissenschaft, 66(3), 302-319. DOI: 10.5771/1615-634X2018-3-302.

Metzger, Miriam J. \& Flanagin, Andrew J. (2013). Credibility and Trust of Information in Online Environments. The Use of Cognitive Heuristics. Journal of Pragmatics, 59, 210-220. DOI: 10.1016/j.pragma.2013.07.012.

Metzger, Miriam J.; Flanagin, Andrew J.; Eyal, Keren; Lemus, Daisy R. \& McCann, Robert M. (2003). Credibility for the 21st Century: Integrating Perspectives on Source, Message, and Media Credibility in the Contemporary Media Environment. Annals of the International Communication Association, 27(1), 293-335. DOI: 10.1080/23808985.2003.11679029.

Metzger, Miriam J.; Hartsell, Ethan H. \& Flanagin, Andrew J. (2015). Cognitive Dissonance or Credibility? A Comparison of Two Theoretical Explanations for Selective Exposure to Partisan News. Communication Research, 47(1), 3-28. DOI: 10.1177/0093650215613136.

Nawratil, Ute (1999). Glaubwürdigkeit als ein Faktor im Prozess medialer Kommunikation. In: Rössler, P. \& W. Wirth (Hrsg.), Glaubwürdigkeit im Internet: Fragestellungen, Modelle, empirische Befunde (15-31). R. Fischer.

Nawratil, Ute (2006). Glaubwürdigkeit in der sozialen Kommunikation. Universitätsbibliothek der Ludwig-Maximilians-Universität München. DOI: 10.5282/ubm/ epub.941.

Neuberger, Christoph (2004). Wandel der aktuellen Öffentlichkeit im Internet.

Neuberger, Christoph (2009). Internet, Journalismus und Öffentlichkeit. In: Neuberger, C.; C. Nuernbergk \& M. Rischke (Hrsg.), Journalismus im Internet (19-105). VS Verlag für Sozialwissenschaften. DOI: 10.1007/978-3-531-91562-3_2.

Neuberger, Christoph \& Quandt, Thorsten (2010). Internet-Journalismus: Vom traditionellen Gatekeeping zum partizipativen Journalismus? In: Schweiger, W. \& K. Beck (Hrsg.), Handbuch Online-Kommunikation (59-79). VS Verlag für Sozialwissenschaften. DOI: 10.1007/978-3-531-92437-3_3.

Newman, Nic; Fletcher, Richard; Kalogeropoulos, Antonis; Levy, David A. L. \& Nielsen, Rasmus Klein (2018). Reuters Institute Digital News Report 2018.

Petty, Richard E. \& Cacioppo, John T. (1986). The Elaboration Likelihood Model of Persuasion. Advances in Experimental Social Psychology, 19, 123-205. DOI: 10.1016/S0065-2601(08)60214-2.

Rimmer, Tony \& Weaver, David (1987). Different Questions, Different Answers? Media Use and Media Credibility. Journalism Quarterly, 64(1), 28-44. DOI: 10.1177/107769908706400104. 
RT Deutsch (2018). Umfrage: Hälfte aller Russen will, dass Putin nach 2024 Präsident bleibt. RT Deutsch, 21. Juni 2018. Abgerufen von https://deutsch.rt.com/ russland/71761-umfrage-halfte-aller-russen-will-putin-nach-2024-als-prasident/.

Sängerlaub, Alexander; Meier, Miriam \& Rühl, Wolf-Dieter (2018). Fakten statt Fakes. Verursacher, Verbreitungswege und Wirkungen von Fake News im Bundestagswahlkampf 2017. Abgerufen von https://www.stiftung-nv.de/sites/default/files/snv fakten_statt_fakes.pdf.

Schmüser, Caroline (2018). Nein - Keine 31 Milliarden Euro an Familienangehörige in Herkunftsländern. Correctiv, 12. Juni 2018. Abgerufen von https://correctiv.org/ faktencheck/2018/06/12/nein-keine-31-milliarden-euro-an-familienangehoerige-inherkunftslaendern/.

Schranz, Mario; Schneider, Jörg \& Eisenegger, Mark (2018). Media Trust and Media Use. In: Otto, K. \& A. Köhler (Hrsg.), Trust in Media and Journalism (73-91). Springer Fachmedien Wiesbaden. DOI: 10.1007/978-3-658-20765-6_5.

Schulze, Tobias (2017). Redaktionsbesuch bei RT Deutsch: Propaganda? Welche Propaganda? taz.de, 22. September 2017. Abgerufen von http://www.taz.de/!5449727/.

Stroud, Natalie Jomini (2014). Selective Exposure Theories. In: Kenski, K. \& K. H. Jamieson (Hrsg.), The Oxford Handbook of Political Communication. DOI: 10.1093/oxfordhb/9780199793471.013.009_update_001.

Sundar, S. Shyam; Knobloch-Westerwick, Silvia \& Hastall, Matthias R. (2007). News cues: Information scent and cognitive heuristics. Journal of the American Society for Information Science and Technology, 58(3), 366-378. DOI: 10.1002/asi.20511.

Tandoc, Edson C. (2019). Tell Me Who Your Sources Are: Perceptions of News Credibility on Social Media. Journalism Practice, 13(2), 178-190. DOI: 10.1080/17512786.2017.1423237.

Tandoc, Edson C.; Lim, Zheng Wei \& Ling, Richard (2018). Defining »Fake News«: A typology of scholarly definitions. Digital Journalism, 6(2), 137-153. DOI: 10.1080/21670811.2017.1360143.

Tsfati, Yariv \& Ariely, Gal (2014). Individual and Contextual Correlates of Trust in Media Across 44 Countries. Communication Research, 41(6), 760-782. DOI: 10.1177/0093650213485972.

Tsfati, Yariv \& Cappella, Joseph N. (2003). Do People Watch what they Do Not Trust? Exploring the Association Between News Media Skepticism and Exposure. Communication Research, 30(5), 504-529. DOI: 10.1177/0093650203253371.

Vallone, Robert P.; Ross, Lee \& Lepper, Mark R. (1985). The Hostile Media Phenomenon: Biased Perception and Perceptions of Media Bias in Coverage of the Beirut Massacre. Journal of Personality and Social Psychology, 49(3), 577-585. DOI: 10.1037/0022-3514.49.3.577.

Voigt, Juliane (2016). Nachrichtenqualität aus Sicht der Mediennutzer. Springer Fachmedien Wiesbaden. DOI: 10.1007/978-3-658-12041-2.

Watergate Redaktion (2018). Finanzminister will 78 Milliarden für Migranten ausgeben. Watergate.tv, 21. Mai 2018. Abgerufen von https://www.watergate.tv/finanz minister-will-78-milliarden-fuer-migranten-ausgeben/. 
Wellbrock, Christian-Mathias (2011). Die journalistische Qualität deutscher Tageszeitungen. Ein Ranking auf Grundlage von Expertenurteilen. Medienwirtschaft, 8(2), 22-31.

Winter, Stephan (2013). Lost in Information? Sozialpsychologische Aspekte der Selektion und Rezeption von journalistischen Online-Angeboten. Kohlhammer Verlag.

Winter, Stephan \& Krämer, Nicole C. (2014). A Question of Credibility. Effects of Source Cues and Recommendations on Information Selection on News Sites and Blogs. Communications, 39(4), 435-456. DOI: 10.1515/commun-2014-0020.

Wohlt, Stephanie; Barkouni, Tarek; Czichy, Anika; Richter, Kirsten; Silge, Kristin \& Welzel, Anna Catharina (2017). »Ich bin ja nicht rechts, aber...«. Eine Untersuchung zum Einfluss einer Bedrohungs- oder Bereicherungsdarstellung auf implizite und explizite Einstellungen gegenüber Flüchtlingen. In: Frindte, W. \& N. Dietrich (Hrsg.), Muslime, Flüchtlinge und Pegida (181-218). Springer Fachmedien Wiesbaden. DOI: 10.1007/978-3-658-17603-7_7.

Zimmermann, Fabian \& Kohring, Matthias (2018). »Fake News« als aktuelle Desinformation. Systematische Bestimmung eines heterogenen Begriffs. Medien \& Kommunikationswissenschaft, 66(4), 526-541. DOI: 10.5771/1615-634X-2018-4-526. 